\title{
POLÍTICA SOCIAL E IGUALDAD DE GÉNERO EN MÉXICO, 2012-2018
}

\author{
SOCIAL POLICY AND GENDER EQUALITY \\ IN MEXICO, 2012-2018
}

\author{
POLITIQUE SOCIALE ET ÉGALITÉ DES SEXES \\ AU MEXIQUE (2012-2018)
}

\author{
Melina Altamirano ${ }^{1}$ \\ El Colegio de México \\ maltamirano@colmex.mx
}

RESUmen: Este artículo explora los vínculos entre la agenda de política social y los objetivos de igualdad de género durante la administración de Enrique Peña Nieto. En particular, me enfoco en la evolución de tres políticas con implicaciones para el bienestar de las mujeres: los servicios de cuidado infantil, el Seguro de Vida para Jefas de Familia y la Pensión para Adultos Mayores. El balance sugiere que, si bien estos esfuerzos favorecieron la atención a mujeres en situación económicamente vulnerable, el énfasis en el combate a la pobreza prevaleció sobre un enfoque basado en derechos sociales. Por tanto, objetivos tales como la equidad de género en la protección social o la socialización de los cuidados y la educación temprana quedaron como asignaturas pendientes. La provisión de estos programas consistió principalmente en transferencias y reprodujo el esquema de fragmentación de las políticas sociales, lo que resultó en bajos niveles de institucionalidad.

Palabras clave: género; mujeres; política social; Estado de bienestar en México

AвSTRACT: This paper explores the links between the social policy agenda and gender equality goals during the Enrique Peña Nieto administration. Specifically, it focuses on the evolution of three policies with implications for the

${ }^{1}$ Agradezco la valiosa asistencia de Berta Díaz Martínez en la realización de esta investigación y el liderazgo de los editores de este número especial. 
wellbeing of women: child care services, life insurance for women heads of households, and pensions for the elderly. The outcome suggests that while these efforts did improve attention to women in vulnerable situations, the emphasis on combating poverty prevailed over one based on social rights. As a result, objectives like gender equity in social protection or the socialization of care and early education remained incomplete. The provisions of these programs principally comprised transfers and repeated the fragmented model of social policies, leading to poor levels of institutionality.

Keywords: gender; women; social policy; welfare state in Mexico.

\section{Traducción de Gonzalo Celorio Morayta}

RÉSumÉ: L'article examine les liens entre l'agenda de la politique sociale et les objectifs d'égalité des sexes sous le gouvernement d'Enrique Peña Nieto. On s'intéresse, en particulier, à l'évolution de trois politiques particulièrement importantes pour le bien-être des femmes: l'offre de soins aux enfants, les assurances-vie des dames chefs de famille et la pension de vieillesse. L'évidence permet de croire que, même si les efforts dans ces domaines ont été utiles aux femmes qui se trouvent dans une situation économique vulnérable, la préférence du gouvernement pour la lutte contre la pauvreté l'a emporté sur une approche privilégiant les droits sociaux. C'est pourquoi des objectifs tels qu'une protection sociale visant à l'égalité des sexes, ou à la socialisation des soins aux enfants et de l'éducation préscolaire, ont été laissés de côté. La mise en ouvre des programmes a surtout consisté à fournir de l'argent, ce qui a aggravé la fragmentation des politiques sociales, aux dépens des institutions. Mots clefs: Genre, femmes, politique sociale, État-providence au Mexique.

Traducción de Bernardo Mabire

Fecha de recepción: junio de 2019

Fecha de aceptación: noviembre de 2019 


\section{A} casi dos décadas de la transición democrática en México, ¿cómo se ha insertado el tema de la igualdad de género en la agenda de política social de la administración federal? El gobierno de Enrique Peña Nieto marcó el regreso del Partido Revolucionario Institucional (PRI) a la presidencia después de dos administraciones panistas en las cuales se dieron transformaciones importantes en el área social, como el Seguro Popular. De manera paralela, la perspectiva de género ha adquirido un lugar más central en la agenda de desarrollo internacional en los últimos años, con implicaciones específicas en las recomendaciones para el diseño de políticas sociales en países latinoamericanos. Este artículo explora las características y el proceso de implementación de tres políticas con claras implicaciones de género: Guarderías y Estancias Infantiles para Apoyar a Madres Trabajadoras; la Pensión para Adultos Mayores (65 y más) y el Seguro de Vida para Jefas de Familia.

Son tres políticas relevantes porque responden, en principio, a necesidades específicas en momentos clave de la vida de la mayoría de las mujeres: la oportunidad de reinsertarse en la fuerza laboral después de la maternidad, la posibilidad de formar un patrimonio y el acceso a una pensión en la vejez.

El texto comienza con una discusión sobre la incorporación del enfoque de género en la agenda de política social, algunas de sus justificaciones teóricas y las implicaciones de esta perspectiva en las políticas públicas. La siguiente sección presenta un panorama breve de la situación de las mujeres en el mercado laboral en México y del esquema de provisión de políticas de bienestar y seguridad social. Posteriormente, se exploran las principales características de las tres políticas, que son el foco del artículo, sus metas y algunos indicadores de afiliación y gasto del gobierno.

El estudio revela la ausencia de una estrategia integral para reducir las brechas de género con herramientas de política social. Si bien se contemplaron metas de inclusión y atención a necesidades específicas de mujeres en situación de vulnerabilidad, no se observa la articulación clara de un 
enfoque de género en el discurso de la administración y en los objetivos de los programas. El esquema de estos programas reprodujo la fragmentación prevalente en el sistema más amplio de provisión de políticas de bienestar en México. Dado que los programas implementados consistieron principalmente en esquemas de transferencias o de subrogación de servicios, el fortalecimiento de la capacidad estatal permaneció en un segundo plano. Objetivos tales como la equidad de género en la protección social o la socialización de los cuidados quedaron también como asignaturas pendientes. En este contexto de provisión fragmentada y con un bajo nivel de institucionalidad, se dificulta la formación de coaliciones ciudadanas amplias que demanden y defiendan las acciones sociales del Estado.

\section{Políticas SOCIALES, GÉNERO Y CURSO DE VIDA}

Los procesos de diseño e implementación de políticas públicas tienen el potencial de atenuar o profundizar las desigualdades de género en la sociedad. Diversos trabajos se han ocupado de estudiar las consecuencias de distintas clases de políticas sociales para una serie de resultados, como la inserción laboral, el acceso a pensiones en la vejez y la capacidad de ahorro, entre otros.

Los regímenes de bienestar de la segunda mitad del siglo xx consideraban a la familia biparental como unidad beneficiaria primaria de la política pública. En este esquema, la persona con la jefatura del hogar adquiría protección mediante su posición en el mercado laboral (formal) y los beneficios se extendían a sus dependientes económicos. Dado que este esquema emergió en contextos donde la participación de la fuerza de trabajo femenina era limitada y existía una marcada división de género entre las esferas privada y laboral, las políticas sociales de conciliación familia-trabajo, por ejemplo, no fueron centrales en el paquete de beneficios so- 
ciales del Estado de bienestar. ${ }^{2}$ Este modelo de familia, denominado en la literatura como de proveedor único masculino, no resulta compatible con dos transformaciones sociales significativas en las últimas décadas: el incremento de la participación laboral de las mujeres y de la proporción de hogares con jefatura femenina. ${ }^{3}$ Estos cambios plantean nuevas presiones en los hogares para conciliar el trabajo de cuidados con el remunerado, lo cual ha motivado reformas al Estado de bienestar en varias democracias. ${ }^{4}$ Dado que el trabajo de cuidados recae principalmente en las mujeres, las políticas sociales tienen un impacto claro en sus opciones laborales y en su capacidad de construir un patrimonio. ${ }^{5}$ Además, en economías con sectores informales amplios, un porcentaje significativo de la población carece de acceso a los sistemas de seguridad social, los cuales suelen contemplar un paquete básico de beneficios para las trabajadoras que incluye, por ejemplo, protección en salud y ahorro para el retiro, además de un piso

2 Para una discusión de los tipos de Estado de bienestar y su intersección con las desigualdades de género, véase Gøsta Esping-Andersen, "A new gender contract", en Gøsta Esping-Andersen (ed.), Why we need a new welfare state, Oxford, University Press, 2002, pp. 68-95.

${ }^{3}$ Véase Nancy Fraser, "After the Family Wage: Gender Equity and the Welfare State”, Political Theory, 22 (1994), pp. 591-618; Diane Sainsbury, Gender, Equality, and Welfare States, Nueva York, Cambridge University Press, 1996, y Chia Liu, Albert Esteve y Rocío Treviño, "Female-headed households and living conditions in Latin America”, World Development, 2017, vol. 90, pp. 311-328.

${ }^{4}$ Para un análisis de las transformaciones a los regímenes de bienestar en favor de la conciliación familia-trabajo, véase Jennifer Pribble, "Mujeres y bienestar: Un estudio comparativo de Chile y Uruguay", en Alma Idiart (ed.), Estado benefactor y políticas sociales: Historia, implementación y reforma de programas sociales en Argentina, Chile y Uruguay, Buenos Aires, Biblos, 2011, pp. 49-76, y Kimberly J. Morgan, "Path shifting of the welfare state: Electoral competition and the expansion of work-family policies in Western Europe”, World Politics, 2013, vol. 65 núm. 1, pp. 73-115.

${ }^{5}$ Brígida García, "El trabajo doméstico y de cuidado: su importancia y principales hallazgos en el caso mexicano", Estudios Demográficos y Urbanos, 34 (2019), pp. 237-267. 
mínimo de programas de conciliación trabajo-familia, como los servicios contributivos de cuidado infantil.

El curso de vida de las personas determina momentos clave que pueden tener efectos duraderos en resultados sociales deseables, como la reducción de las desigualdades económicas y de género. Las políticas sociales pueden ser herramientas esenciales para proteger el ingreso y amortiguar el efecto de los sucesos cruciales de vida sobre la trayectoria de las personas. Para las mujeres, los riesgos sociales específicos se entrelazan de manera importante con la reproducción y la carga del trabajo de cuidados. Por ejemplo, se ha encontrado evidencia de que entre las mujeres jóvenes que no estudian ni tienen un trabajo remunerado en América Latina, el tiempo dedicado a tareas de cuidado en el hogar es mayor. ${ }^{6}$ La etapa reproductiva es un momento determinante, pues las mujeres son más propensas a reducir sus horas de trabajo o a abandonar la fuerza laboral remunerada después del nacimiento de un hijo. ${ }^{7}$ Una serie de trabajos han señalado la importancia de considerar las trayectorias de vida diferenciadas de las mujeres en el diseño de las políticas sociales para incidir en estos momentos clave, por ejemplo, socializando el trabajo de cuidados o amortiguando el efecto de las ausencias del mercado laboral. ${ }^{8}$ Los estudios al respecto han mostrado que dichas ausencias y la separación del trabajo remunerado tienen un impacto negativo en el monto de las cotizaciones a la seguridad social de las mujeres. Para aquellas incorporadas al sector formal, las interrupciones en la trayectoria laboral derivan en un menor número de años de contribuciones a la seguridad social,

${ }^{6}$ Rafael Novella et al. (eds.), Millennials en América Latina y el Caribe: ¿trabajar o estudiar?, Washington, CEEY - Banco Interamericano de Desarrollo, 2018, http://dx.doi.org/10.18235/0001410

${ }^{7}$ Brígida García y Edith Pacheco, Uso del tiempo y trabajo no remunerado en México, México, El Colegio de Mexico, 2014.

${ }^{8}$ Véase Janet C. Gornick y Marcia K. Meyers, "Creating gender egalitarian societies: An agenda for reform”, Politics E Society, 36 (2008), pp. 313-349. 
mientras que la transición a puestos de tiempo parcial a menudo implica una merma en el salario base de cotización. ${ }^{9}$ Por tanto, al considerar los obstáculos específicos y las necesidades diferenciadas de las mujeres en etapas cruciales, la atención al curso de vida en la definición de las políticas sociales tiene el potencial de contrarrestar la acumulación y reproducción de las desigualdades sociales.

\section{MUJERES, MERCADO LABORAL Y LA FRAGMENTACIÓN DE LA PROTECCIÓN SOCIAL}

En México, el esquema de incorporación a la seguridad social siguió desde sus inicios el modelo del proveedor único. ${ }^{10} \mathrm{El} \mathrm{ac}-$ ceso a la seguridad social está condicionado a la obtención y permanencia en un empleo formal. Una vez afiliados al sistema, los trabajadores tienen la posibilidad de asegurar a sus dependientes económicos, quienes se vuelven derechohabientes, por ejemplo, de los servicios de salud del Instituto Mexicano del Seguro Social (Imss) o del Instituto de Seguridad y Servicios Sociales de los Trabajadores del Estado (ISSSTE). ${ }^{11}$ En este esquema, la incorporación de las necesidades de conciliación trabajo-familia partió del supuesto de que, en la mayoría de los casos, los empleados con dependientes económicos menores de edad son parte de hogares biparentales, en los que la pareja (mujer) es la cuidadora principal de

${ }^{9}$ María Luisa González Marín et al., "Las mujeres y los nuevos sistemas de pensiones en México”, Revista de Investigación Social, 4 (2007), pp. 109-133.

10 Michelle Dion, "Retrenchment and Reform", en su libro Workers and welfare: Comparative institutional change in twentieth-century Mexico, Pittsburgh, University of Pittsburgh Press, 2010, p. 116-151.

11 Otras instituciones de gobierno que proporcionan esquemas de aseguramiento similares a sus trabajadores son los institutos para trabajadores de gobiernos estatales, de Petróleos Mexicanos y de otras dependencias gubernamentales. 
los hijos. ${ }^{12}$ Por tanto, el acceso a los servicios de cuidado infantil de las instituciones de seguridad social estuvo pensado como una prestación útil exclusivamente para las mujeres trabajadoras y sólo otorgada en circunstancias extraordinarias a los hombres (por ejemplo, en caso de viudez). ${ }^{13}$

A la incompatibilidad del modelo de protección de las instituciones de seguridad social con las transformaciones recientes de los hogares mexicanos se suma su limitada cobertura. Más de la mitad de la fuerza laboral en el país está inserta en el sector informal. ${ }^{14}$ Dada la segmentación en el tipo de servicios, según el tipo de trabajo (formal o informal) de las personas, los derechos sociales, las prestaciones laborales y el impacto del esfuerzo de política social del Estado mexicano están condicionados al estatus laboral. La estructura del mercado de empleo mexicano implica que una elevada proporción de trabajadores no cuente con cobertura de salud, un esquema de ahorro para el retiro y vivienda ni, por consiguiente, acceso a servicios de conciliación traba-

${ }^{12}$ Esta serie de supuestos implica también una concepción de pareja exclusivamente heterosexual. La movilización de grupos de la comunidad LGBTT+ en México ha generado transformaciones cruciales de estas visiones restrictivas de cómo se forman los hogares. En noviembre de 2018, el Senado de la República aprobó una reforma para garantizar que cónyuges de asegurados/as de parejas del mismo sexo tengan acceso a las prestaciones de seguridad social del IMss y del issste.

${ }^{13}$ Después de una serie de litigios de padres en demanda de acceso igualitario al servicio de guarderías como derechohabientes del IMss, en abril de 2019, la Cámara de Diputados aprobó una reforma a la ley del Seguro Social para extender el servicio de cuidado infantil a los padres derechohabientes de dicha institución.

${ }^{14}$ La tasa de informalidad laboral (TIL 1) estimada por el Instituto Nacional de Estadística y Geografía (INEGI) para diciembre de 2018 fue de $56.8 \%$ de la población ocupada en México. Esta cifra corresponde a "la suma, sin duplicar, de los que son laboralmente vulnerables por la naturaleza de la unidad económica para la que trabajan, con aquellos cuyo vínculo o dependencia laboral no es reconocido por su fuente de trabajo". Fuente: INEGI, "Indicadores de ocupación y empleo. Cifras oportunas durante diciembre de 2018", 22 de enero de 2019, https://www.inegi.org. $\mathrm{mx} /$ contenidos/saladeprensa/boletines/2019/iooe/iooe2019_01.pdf 
jo-familia, como las guarderías. Para las mujeres que enfrentan obstáculos específicos por la carga de los cuidados y el impacto de los sucesos en su curso de vida, la informalidad supone un nivel exacerbado de vulnerabilidad. ${ }^{15} \mathrm{El} 57.2 \%$ de las mujeres que trabajan en áreas urbanas de México se encuentran en el sector informal. ${ }^{16}$

La participación de las mujeres en el trabajo remunerado es un indicador fundamental de igualdad de género. Según datos de la Organización para la Cooperación y el Desarrollo Económicos (OCDE), en México, sólo el 47.3\% de las mujeres entre 15 y 64 años tiene un empleo remunerado. ${ }^{17}$ Esta tasa de participación está por debajo del promedio de América Latina y es la más baja en el listado de la ocDe, sólo por encima de Turquía. La ocupación en el mercado laboral está asociada con la posibilidad para las mujeres de alcanzar independencia financiera y contribuir a una cuenta de ahorro para el retiro. Además, los hogares con doble ingreso tienen

15 Algunos estudios sugieren que los empleos en el sector informal podrían ser particularmente atractivos para las mujeres con hijos, quienes optarían por trabajar en este sector dada su mayor flexibilidad para conciliar el trabajo productivo y de cuidado. No obstante, esta decisión de algunas mujeres de emplearse informalmente por lo común es producto de la alta incompatibilidad de los requerimientos de los empleos formales (casi siempre de baja calificación) con las necesidades de cuidado de los hijos. Para un debate sobre el empleo informal y la flexibilidad laboral, véase Danielle L. Rodin et al., "Determinants of informal employment among working mothers in Mexico”, Community, Work EF Family (2012), núm. 1, pp. 85-99. También, Guillermo E. Perry et al., Informality: Exit and exclusion, Washington, DC, World Bank Publications, 2007.

16 Porcentaje de mujeres ocupadas en condición de informalidad, mujeres de 25 a 54 años en 2014. Mariana Marchionni et al., Participación laboral femenina, ¿qué explica las brechas entre países?, IDB-CEDLAS, Nueva York, 2019. http://dx.doi.org/10.18235/0001512

17 Organisation for Economic Co-operation and Development, OECD, OECD.Stats, https://stats.oecd.org/index.aspx?qu eryid=54741. El INEGI reporta que en el primer trimestre de 2019, 45 de cada 100 mujeres eran económicamente activas. Véase InEgi, Encuesta Nacional de Empleo, mayo de 2019, https://www.inegi.org.mx/contenidos/saladeprensa/bole tines/2019/enoe_ie/enoe_ie2019_08.pdf 
una mayor probabilidad de situarse por encima de la línea de pobreza y son menos vulnerables a la pérdida del empleo de alguno de los miembros de la familia. ${ }^{18}$ Cuando en ésta hay hijos u otros menores dependientes, la tasa de participación femenina refleja de manera agregada las decisiones que se toman en los hogares, las cuales a menudo consideran estrategias de conciliación trabajo-cuidado. Estas decisiones a nivel individual y del hogar están a su vez condicionadas por la estructura del mercado laboral, el nivel de acceso y las características de las políticas sociales. Hay estudios que muestran, por ejemplo, que el disfrute de servicios de cuidado infantil aumenta la probabilidad de que las mujeres se integren a la fuerza laboral. ${ }^{19}$

\section{LA AGENDA DE POLÍTICA SOCIAL, 2012-2018}

Si bien el regreso del PRI al gobierno federal en 2012 trajo consigo algunos cambios en la agenda social, hubo una clara continuidad en el paradigma de provisión de los principales servicios y programas. Dos elementos centrales de la estrategia de política social se mantuvieron en el periodo 2012-2018: la preponderancia de los programas no contributivos y el reforzamiento de un modelo fragmentado de provisión con diversos beneficios, poblaciones objetivo y requisitos de afiliación.

La primera característica de la estrategia social en las últimas décadas ha sido el papel de las transferencias (particularmente las focalizadas y condicionadas) como instrumento de política social predominante. Luego de la creación de las instituciones de seguridad social, las transformaciones más

${ }^{18}$ Para una análisis sobre las implicaciones de la participación laboral femenina en el ingreso en los niveles de pobreza y desigualdad, véase Leonardo Gasparini y Mariana Marchionni, Bridging gender gaps? The rise and deceleration of female labor force participation in Latin America, La Plata, CEDLAS, 2015.

${ }^{19}$ Véase Gabriela Calderón, "The Effects of Child Care Provision in Mexico", México, documento de trabajo 2014-07, Banco de México, 2014. 
notables en el esquema de la política social en el país se dieron en las últimas administraciones priistas, antes de la alternancia. Ante las presiones para ampliar la cobertura de servicios a la población abierta no derechohabiente, el Programa de Desarrollo Humano Oportunidades/Prospera Programa de Inclusión Social, de transferencias condicionadas, se consolidó como un modelo de prestaciones a nivel regional que atendía las metas de reducción de la pobreza con énfasis en la generación de capital humano. Este modelo se mantuvo después de la llegada de los gobiernos panistas a la administración federal y durante el sexenio de Enrique Peña Nieto.

La segunda característica tiene que ver con el reforzamiento de la fragmentación del sistema, el cual ha generado nuevos programas que se suman al modelo existente y que implican múltiples políticas sociales con distintas poblaciones objetivo, beneficios variados y umbrales diferenciados de requisitos de afiliación. Así, mientras los servicios y beneficios para la población adscrita a las instituciones de seguridad social cambiaron relativamente poco en el periodo, la administración del presidente Peña Nieto continuó con la estrategia de implementación de nuevos programas para la población no derechohabiente y con los esfuerzos no contributivos emprendidos por las administraciones panistas. ${ }^{20}$ Como veremos más adelante, la justificación de la estrategia de política social del gobierno federal de 2012-2018 fue, de manera crucial, el combate a la pobreza.

${ }^{20}$ Para un análisis de la política social en el sexenio del presidente Calderón, véase Laura Flamand y Carlos Moreno-Jaimes, "La protección social en salud durante el gobierno de Calderón. Avances y rezagos en el diseño y la implementación del Seguro Popular (2006-2012)”, Foro Internacional 55 (2015), núm. 1, pp. 217-261. Para un estudio de los cambios en la agenda social durante los primeros años del sexenio del presidente Peña Nieto, véase Laura Flamand, "La reforma perdida. Tendencias de la política social en México (2012-2015)”, en Fernando Nieto y Ernesto Velasco (eds.), Una agenda para la administración pública: reconocimiento a la trayectoria de María del Carmen Pardo, México, El Colegio de México, 2018. 
Los ajustes e innovaciones a los programas sociales insignia de la administración se refirieron principalmente a la necesidad de abatir los rezagos de las comunidades en situación de pobreza y de los hogares con un nivel elevado de vulnerabilidad. Una implicación importante de este enfoque fue su prevalencia sobre una justificación basada en derechos sociales. El paradigma de la política social en la administración 2012-2018 se reflejó en las características de los programas y restringió su articulación en un marco más integral de abatimiento de las desigualdades de género.

Finalmente, si bien al inicio del periodo hubo un impulso importante a la estrategia social del gobierno federal, las prioridades cambiaron hacia el final del sexenio. El presupuesto presentado en 2014 contempló incrementos significativos en el monto para políticas sociales, especialmente para los programas Seguro de Vida para Jefas de Familia (150\%) y la Pensión para Adultos Mayores $(74 \%) .{ }^{21}$ En contraste, la asignación de recursos se redujo notablemente en 2016, lo cual restringió los alcances de los esfuerzos en política social.

\section{LAS MUJERES EN LA AGENDA SOCIAL DURANTE el gobierno de Peña Nieto}

En un acto público, el 9 de mayo de 2014, el presidente Peña Nieto señaló que el Plan de Desarrollo 2013-2018 incluía, por primera vez, una perspectiva de género "para incorporar las necesidades y prioridades de las mujeres en todas las acciones del gobierno". ${ }^{22}$ En particular, el presidente hizo referencia a tres de las políticas sociales implementadas durante la administración 2012-2018 como evidencia del enfoque de género de la agenda social: las Guarderías y Estancias Infan-

21 Itxaro Arteta, "Priorizan para 2014 programas sociales", Reforma, 10 de septiembre de 2013.

22 "Peña Nieto anuncia plan de vivienda para jefas de familia", Notimex, 9 de mayo de 2014. 
tiles para Apoyar a Madres Trabajadoras, la Pensión para Adultos Mayores (también conocida como 65 y Más) y el Seguro de Vida para Jefas de Familia.

En efecto, por su diseño y poblaciones objetivo, estos programas atendieron vulnerabilidades asociadas a la maternidad, la tercera edad y la potencial desprotección de menores dependientes en caso de ausencia de la madre. A continuación, se presenta un panorama general de las características y evolución de estos tres programas. El programa de pensiones no contributivas 65 y Más y el Seguro de Vida fueron componentes principales de la estrategia antipobreza del gobierno de Peña Nieto, la cual ocupó un lugar prioritario en el discurso de su administración durante los primeros años. ${ }^{23}$ El programa de Guarderías y Estancias Infantiles, un legado de la segunda presidencia panista, tuvo menos visibilidad durante el sexenio de Peña Nieto. Sin embargo, se mantuvo este esquema de servicios de cuidado infantil como un componente de la estrategia social del gobierno federal y se usó como referente de las acciones sociales orientadas a ampliar las oportunidades de mujeres en situación de vulnerabilidad.

Como veremos, si bien estos esfuerzos tenían el potencial de articularse en una agenda integral de reducción de las desigualdades económicas y de género, el paradigma predominante de la política social limitó sus alcances, por lo que permanecieron como programas antipobreza para individuos y hogares.

\section{Estancias infantiles para madres trabajadoras}

Una de las reformas más importantes en materia de políticas de cuidado infantil en México fue la introducción, en 2007,

${ }^{23}$ Durante 2013 y 2014, el presidente Peña Nieto puso énfasis en varias ocasiones en el objetivo del abatimiento de la pobreza como la prioridad nacional. Antonio Baranda, "Alista Peña Nieto plan antipobreza", Reforma, 10 de enero de 2013. 
del servicio público de guarderías a población no derechohabiente, durante el gobierno del presidente Felipe Calderón. El programa Guarderías y Estancias Infantiles para Apoyar a Madres Trabajadoras fue creado con el objetivo de ofrecer servicios de cuidado infantil a las mujeres que trabajan en el sector informal y que, por tanto, no tienen acceso a las guarderías que forman parte de las instituciones de seguridad social como el IMss y el IsssTe. La iniciativa, administrada por la Secretaría de Desarrollo Social (Sedesol), estableció como requisitos de afiliación tener un ingreso igual o menor a seis salarios mínimos y no ser derechohabiente de instituciones de seguridad social. ${ }^{24}$ La provisión de los servicios de cuidado recayó en guarderías privadas, en un esquema que otorgaba un apoyo inicial de hasta 35000 pesos destinado a la adaptación de espacios para volverlos estancias infantiles, a la compra del material e insumos necesarios para el cuidado de los menores y a la capacitación del personal administrativo y operativo. El apoyo de inversión se entregaba directamente a individuos que hacían una solicitud formal a Sedesol para participar en el programa como dueños de estancias. El programa establecía pagos mensuales posteriores a cada una de acuerdo con el número de menores inscritos en el esquema de Guarderías y Estancias Infantiles. ${ }^{25}$ Una vez afiliadas, las beneficiarias (y padres beneficiarios) recibían un apoyo de hasta 700 pesos mensuales para complementar el pago por el cuidado de sus hijos menores.

La puesta en marcha de las Guarderías y Estancias Infantiles permitió ampliar notablemente la cobertura nacional

${ }^{24}$ Reglas de Operación del Programa de Guarderías y Estancias Infantiles para Apoyar a Madres Trabajadoras, para el ejercicio fiscal 2007, Diario Oficial de la Federación, 10 de enero de 2007.

25 Se estableció un subsidio por "una cantidad fija de $\$ 350$ pesos por 11 días de servicio, más una cantidad variable proporcional a los días de asistencia de cada niño o niña, equivalente a $\$ 87.50$ pesos diarios”, con un monto mayor destinado al cuidado de los menores con algún tipo de discapacidad, loc. cit. A lo largo del periodo 2012-2018 estos montos se ajustaron anualmente para incorporar el efecto de la inflación. 
de servicios de cuidado hacia el final del sexenio de Felipe Calderón, con un monto significativamente menor de inversión por niño inscrito en comparación con los servicios de guardería en instituciones de seguridad social. La participación de entes privados en la provisión del servicio significó que el personal administrativo y operativo de las estancias no tenía una relación contractual con el gobierno federal. Para monitorear el estado de las instalaciones y las condiciones del servicio, personal del Sistema Nacional para el Desarrollo Integral de la Familia (DIF) realizaban visitas periódicas para reportar a Sedesol el cumplimiento de los criterios de permanencia establecidos en las reglas de operación del programa.

La administración de Enrique Peña Nieto mantuvo este programa y lo integró a su agenda central de combate a la pobreza. La gráfica 1 muestra el número de niñas y niños inscritos en estancias de Sedesol de 2012 a 2017. En general, se observa una tendencia estable en el número de menores afiliados al programa. Después de una disminución de 3\%, de 2012 a 2013, la cobertura aumentó a 515 795, en 2015, y se mantuvo en alrededor de medio millón de niñas y niños inscritos en el resto del sexenio. ${ }^{26}$

$\mathrm{Al}$ igual que en el caso de Oportunidades/Prospera, otro legado de política social de gobiernos previos, el discurso oficial resaltó los aspectos positivos del diseño del programa Guarderías y Estancias Infantiles, enfatizando los beneficios de su continuación. Sin embargo, hubo un esfuerzo limitado para enriquecer el programa con otros componentes visibles o para vincularlo con otras políticas sociales en el ámbito educativo o laboral. La motivación y lógica del programa conti-

${ }^{26}$ El nivel de cobertura, sin embargo, permaneció bajo en relación con el total de la población menor de tres años. Según datos de la Organización para la Cooperación y el Desarrollo Económicos (OCDE), el porcentaje de niños y niñas de 0 a 2 años inscritos en servicios de cuidado infantil en 2017 era de $3.7 \%$. El promedio de los países miembros de la oCDE era de $35 \%$ para este rango de edad. Véase oECD, “OECD Family Database", http://www.oecd.org/els/family/database.htm 
nuó orientada a la atención a la población económicamente vulnerable para facilitar la inserción o reinserción de mujeres de bajos recursos en el mercado de trabajo.

\section{GRÁfICA 1}

Niños y niñas inscritos en estancias infantiles de Sedesol (2012-2017)

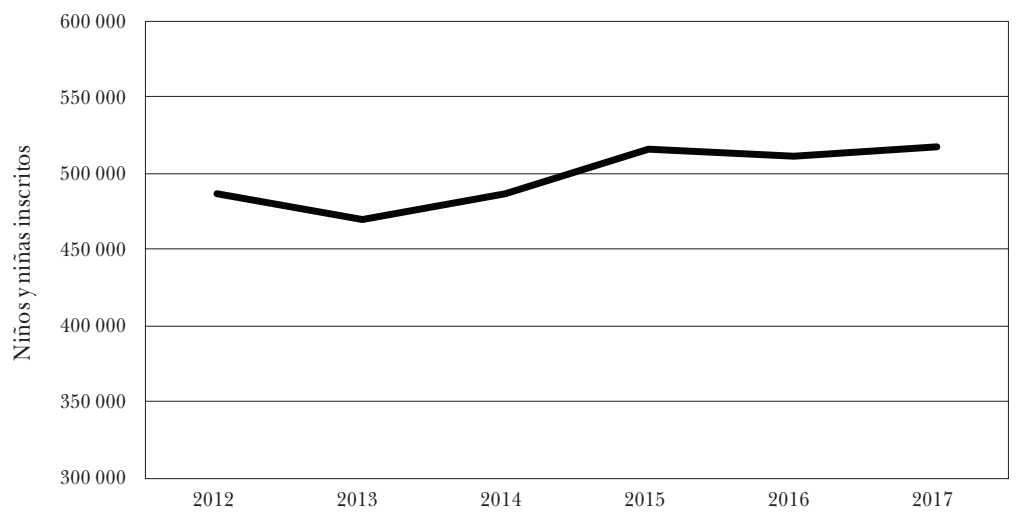

Fuente: elaboración propia con base en la respuesta a la solicitud de información a Sedesol 0002000129118.

La gráfica 2 muestra el monto del presupuesto ejercido en el mismo periodo. Los datos revelan un esfuerzo presupuestario estable en la asignación de recursos a lo largo del sexenio, con un promedio de alrededor de 3829 millones de pesos anuales en el periodo 2012-2017. Esta asignación fija de recursos garantizó la operación del programa durante la administración del presidente Peña Nieto, pero no reflejó un esfuerzo por ampliar su cobertura o invertir más recursos en la capacitación del personal de las estancias. Si bien en 2016 se anunció un acuerdo entre la Secretaría de Educación Pública (SEP) y Sedesol para ofrecer educación preescolar a partir de los cuatro años en las estancias, el avance para 
establecer lineamientos educativos en edades más tempranas fue modesto. ${ }^{27}$

Por otro lado, durante el sexenio perduraron las brechas en el acceso y en las características de la prestación entre los servicios de cuidado infantil contributivos y no contributivos. A pesar de que en el periodo las estancias de Sedesol incorporaron a más niños y niñas que las guarderías para derechohabientes de instituciones de seguridad social, el presupuesto asignado al sistema contributivo fue más elevado. ${ }^{28}$ La continuación de este esquema fragmentado reforzó las inequidades en la calidad de ambos sistemas de provisión de cuidado infantil. ${ }^{29}$

27 Por ejemplo, en la revisión que hizo la Auditoría Superior de la Federación (ASF) al programa en 2017, se señalaron insuficiencias en la acreditación de las capacitaciones complementarias del personal convocadas por el DIF nacional y en la matriz de indicadores de metas/resultados. En el transcurso de la auditoría a partir de las peticiones de la ASF, la Sedesol elaboró mecanismos de control y una nota metodológica para acreditar estos criterios de evaluación. Véase Auditoría Superior de la Federación, "Informe Individual del Resultado de la Fiscalización Superior de la Cuenta Pública 2017, Auditoría de Desempeño: 2017-0-20100-070265-2018", 2017, https:/ /www.asf.gob.mx/Trans/Informes/IR2017a/Do cumentos/Auditorias/2017_0265_a.pdf

${ }^{28}$ En 2017, el número de menores inscritos en guarderías del IMss y estancias del IssSTe fue 227022 en total. Es decir, en conjunto, el sistema contributivo correspondió al $30.5 \%$ de la población infantil incorporada a la red de estancias de Sedesol. Véase Memoria estadística del IMss 2017, http:/ / www.imss.gob.mx/conoce-al-imss/memoria-estadistica-2017 y Anuario estadístico del IsssTe de 2017, http://www.issste.gob.mx/datosabiertos/anuarios/anuarios2017.html En contraste, el presupuesto conjunto ejercido por el sistema IMss e ISSSTE de cuidado infantil en el mismo año representó el 332\% de los recursos ejercidos en la red de estancias infantiles de Sedesol. Fuente: solicitudes de información a Sedesol, IMss e IssSTE (0002000129118/0063700452018/0064101991818).

${ }^{29}$ Cabe señalar que aun con el nivel de inversión y margen de mejora del programa Guarderías y Estancias Infantiles, las evaluaciones de impacto en el estatus laboral de los padres apuntan a una mayor inserción laboral femenina e incluso en el nivel de remuneración de los padres. Estudios recientes sugieren un efecto positivo del tiempo de los niños en dicho programa sobre su desarrollo cognitivo. Véase Guillermo M. Cejudo et al., "Metaevaluación del Programa de Estancias Infantiles (PEI)", 


\section{GRÁFICA 2}

Presupuesto ejercido por el programa Guarderías y Estancias Infantiles para Apoyar a Madres Trabajadoras, de Sedesol (2012-2017)

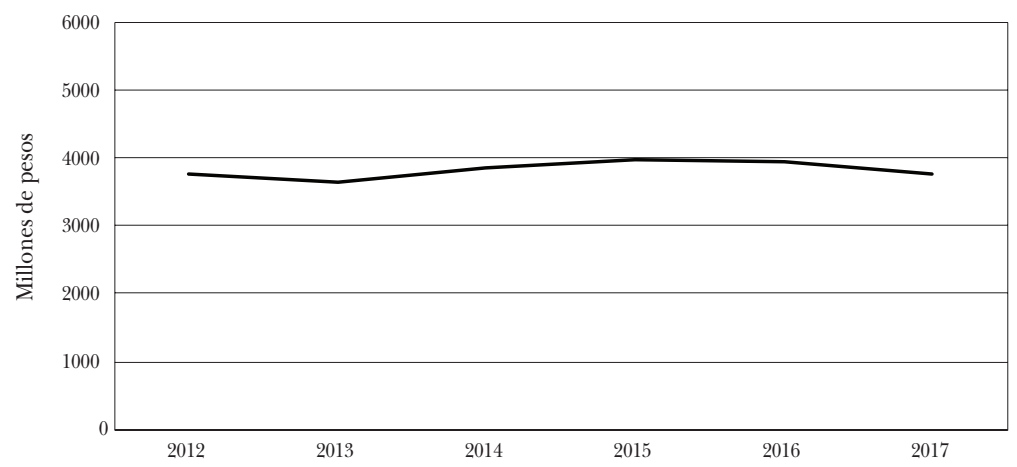

Fuente: elaboración propia con base en la respuesta a la solicitud de información a Sedesol 0002000129118. Valores a precios de 2017.

Finalmente, la baja institucionalidad del programa desfavoreció la formación de una coalición amplia que impulsara la mejora de los servicios de cuidado infantil no contributivos. Las estancias infantiles de Sedesol permanecieron como una red de establecimientos administrados de manera independiente, sin intervención directa del Estado. Este modelo posiblemente dificultó una atribución clara de responsabilidades gubernamentales para las madres (y padres) con hijos en estancias infantiles, y la posibilidad de identificarse y organizarse como beneficiarias de la misma política pública de cuidado infantil.

México, cide-clear, 2012; Antonio Rizzoli-Córdoba et al., "Asociación entre el tiempo de permanencia en el Programa de Estancias Infantiles para niños en situación de pobreza y el nivel de desarrollo infantil”, Boletín Médico del Hospital Infantil de México, 2017, núm. 2, pp. 98-106; G. Calderón, op. cit. 
Pensión para Adultos Mayores

El programa federal de transferencias para adultos mayores se inició en 2007 bajo el nombre Programa de Atención a los Adultos Mayores de 70 años y Más en Zonas Rurales. Este programa se sumó al esquema de pensiones contributivo que, para entonces, había transitado a un sistema de cuentas de ahorro individuales de los trabajadores en el sector formal. ${ }^{30}$ Siguiendo el modelo del programa de transferencias implementado en la Ciudad de México desde 2003, el gobierno de Felipe Calderón puso en marcha los apoyos para Atención a los Adultos Mayores de 70 años y Más y expandió gradualmente su cobertura a lo largo del sexenio. ${ }^{31}$

La administración de Peña Nieto mantuvo el programa orientado a la población de la tercera edad sin acceso a pensiones contributivas y redujo la edad mínima para ser beneficiario de 70 a 65 años en 2013. El nombre de este programa federal cambió a Pensión para Adultos Mayores (también publicitado como 65 y Más). Se establecieron transferencias bimestrales de 1050 pesos para depositarse automáticamente en una tarjeta individual. El gobierno federal presentó estos cambios al programa en el marco de su estrategia insignia de combate a la pobreza, el Sistema Nacional para la Cruzada contra el Hambre. Según las reglas de operación de 65 y Más, los objetivos de estas transferencias eran "contribuir a la reducción de la vulnerabilidad de la población adulta mayor de 65 años en adelante que no recibe ingresos por concepto de pago de jubilación o pensión de tipo contributivo, mediante la entrega de apoyos económicos y de protección social". ${ }^{32}$

${ }^{30}$ Véase M. Dion, op. cit., cap. 5, y Miguel Ángel Yunes, La reforma del ISSSTE: un cambio necesario, México, FCE, 2009.

31 Véase L. Flamand, op. cit.

${ }^{32}$ Acuerdo por el que se emiten las Reglas de Operación del Programa de Pensión para Adultos Mayores, para el ejercicio fiscal 2013, Diario Oficial de la Federación, 20 de febrero de 2013. 
En 2013, se anunció que se daría prioridad en la asignación de recursos del programa a la población objetivo establecida en el plan de la Cruzada contra el Hambre. ${ }^{33}$ El encuadre de 65 y Más en la estrategia antipobreza del gobierno federal favoreció su continuación y expansión como una política coherente con la agenda de desarrollo de la administración de Peña Nieto. No obstante, el enfoque exclusivo en el combate a la pobreza relegó la atención a la desigualdad de género en el curso de vida y su potencial para mejorar el diseño y la implementación de las pensiones no contributivas.

Si bien el abatimiento de la pobreza es una justificación crucial para el impulso de políticas sociales orientadas a los adultos mayores, este enfoque constituye un piso mínimo. Los programas no contributivos para la tercera edad tienen implicaciones especialmente relevantes para el bienestar de las mujeres adultas mayores, incluyendo el de aquéllas en hogares por encima de la línea de bienestar. Dada la inserción desigual de las mujeres en el mercado de trabajo, el acceso a pensiones contributivas presenta claras disparidades de género. Según estimaciones de 2018 de la Organización Internacional del Trabajo (оIт), mientras que $33.3 \%$ de los hombres de 65 años en México tiene un ingreso vía una pensión contributiva, tan solo $9.5 \%$ de las mujeres cuenta con esta protección. ${ }^{34}$ Por otro lado, la esperanza de vida para las mujeres en México es mayor que la de los hombres, lo cual implica que las mujeres enfrentan periodos más prolongados de desprotección social en los últimos años de vida. ${ }^{35}$

La gráfica 3 presenta la evolución del número de beneficiarios del programa, según sexo, de 2013 a 2018. Los datos

33 Itxaro Arteta, “Alinean proyecto con 65 y Más”, Reforma, 19 de febrero de 2013.

34 Organización Internacional del Trabajo, Presente y futuro de la protección social en América Latina y el Caribe, Lima, OIT, Oficina Regional para América Latina y el Caribe, 2018, p. 97.

${ }^{35}$ En 2018, la esperanza de vida para las mujeres era de 78.1 años y para los hombres de 73. Instituto Nacional de Estadística y Geografía, Mujeres y hombres en México 2018, México, INEGI, 2018. 
muestran un incremento en el número de adultos mayores afiliados entre 2013 y 2015, durante los años del impulso inicial a la estrategia antipobreza. La tendencia es más marcada en el caso de las mujeres. Además, la gráfica revela una mayor presencia femenina entre los beneficiarios de las transferencias del programa 65 y Más. De los cinco millones promedio de adultos mayores afiliados al programa en el periodo, en promedio, tres fueron mujeres y dos, hombres. Estas diferencias apuntan a la necesidad de vincular el programa con otras políticas sociales que atiendan las necesidades específicas de las mujeres de la tercera edad, como la protección de la salud. Si bien durante el sexenio se anunciaron acciones orientadas a complementar el programa con servicios de acompañamiento geriátrico y específico en salud, estas iniciativas no se concretaron en una expansión visible de los alcances del programa.

\section{GRÁFICA 3}

Número de beneficiarios del programa Pensión para Adultos Mayores, según sexo (2013-2017)

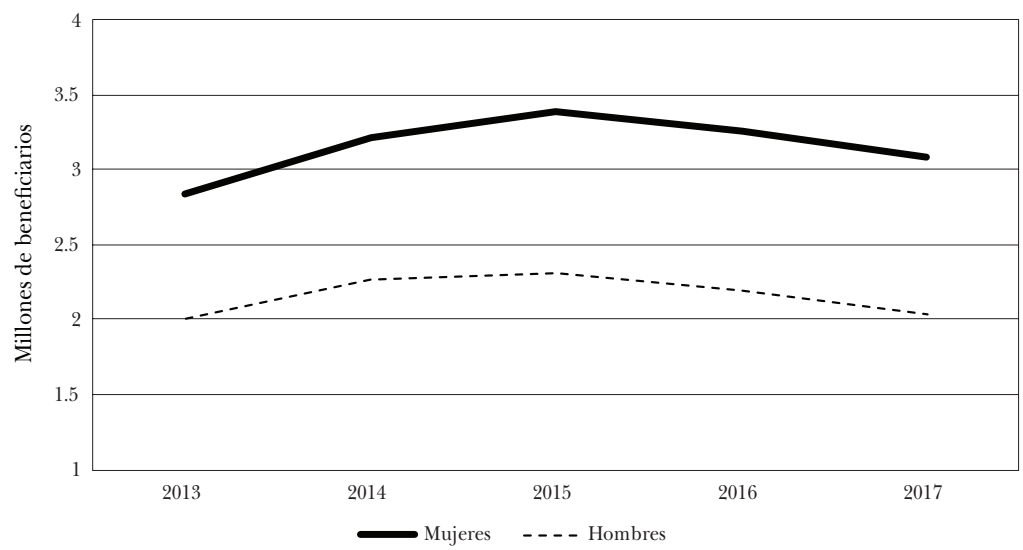

Fuente: elaboración propia con base en fichas de monitoreo y evaluación anual de Coneval. 
La gráfica 4 muestra la tendencia del presupuesto asignado al programa entre 2013 y 2018. Después del impulso inicial a la estrategia de la Cruzada contra el Hambre (2013-2015), los recursos asignados para 65 y Más disminuyeron paulatinamente en el resto del sexenio. En 2018, el programa recibió un presupuesto $22.3 \%$ menor que lo asignado en 2014. Esta reducción pudo haber estado relacionada con los ajustes a los niveles de gasto social al cierre del sexenio y también puede reflejar los cambios en el número de adultos mayores afiliados al programa.

GrÁFICA 4

Presupuesto asignado al programa Pensión para Adultos Mayores (2013-2018)

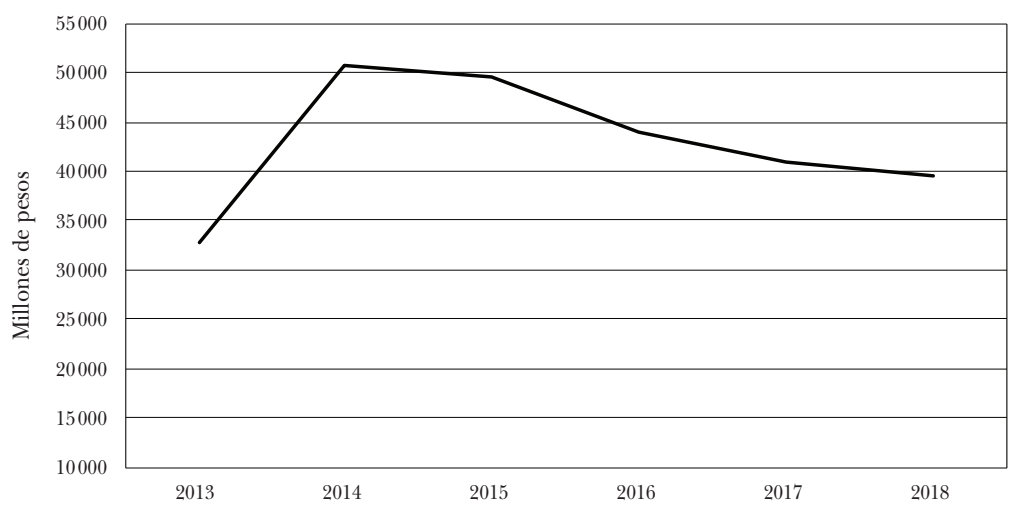

Fuente: elaboración propia con datos de la Secretaría de Desarrollo Social (Sedesol). Información obtenida vía IPRO, "Iniciativa para el fortalecimiento de la institucionalidad de los programas sociales", https:// www.programassociales.org.mx/ (valores a precios constantes de 2018).

En suma, las transferencias a adultos mayores se consolidaron como modelo de política social, el cual incluso se expandió al reducir la edad de afiliación. No obstante, el programa estuvo sujeto a fluctuaciones en las prioridades de gasto. Ade- 
más, para asegurar la efectividad de este esfuerzo para favorecer el bienestar de los adultos mayores, sería necesario reunir evidencia sobre el impacto de las transferencias en su calidad de vida.

Los estudios disponibles sugieren que las transferencias a adultos mayores pueden modificar los patrones de gasto y consumo de los hogares, a veces con resultados no esperados si se canalizan a inversiones en otros miembros de la familia. ${ }^{36}$ Para ello, será crucial vincular el programa con otras políticas, como las de protección de la salud para la tercera edad.

\section{Seguro de Vida para Jefas de Familia}

Finalmente, uno de los programas sociales que tuvieron mayor visibilidad durante la administración fue el Seguro de Vida para Jefas de Familia, que se inició en el primer trimestre de 2013.

El presidente Peña Nieto lo presentó como uno de los tres programas sociales rectores de su administración (junto con 65 y Más y la Cruzada contra el Hambre). ${ }^{37} \mathrm{El}$ programa buscó atender a un segmento de hogares particularmente

${ }^{36}$ Por ejemplo, Gutiérrez, Juárez y Rubli encuentran que las transferencias del programa local para adultos mayores en la Ciudad de México incrementan la probabilidad de que los niños en el hogar se incorporen a la escuela. Véase Emilio Gutiérrez et al., "The Effect of a Transfer Program for the Elderly in Mexico City on Co-Residing Children's School Enrollment", World Bank Economic Review, 31 (2017), pp. 809-828. Águila, López-Ortega y Gutiérrez Robledo muestran que la periodicidad de la entrega de las transferencias tiene un efecto en la salud de los adultos mayores. La entrega bimestral no parece elevar el bienestar físico de los beneficiarios (contrariamente a la transferencia mensual), lo cual sugiere que al menos parte del ingreso por el programa se destina a aspectos no relaciondos con la salud de los adultos mayores, especialmente en el caso de las mujeres. Véase Emma Águila et al., "Non-contributory pension programs and frailty of older adults: evidence from Mexico", PLOS One (2018), núm. 11, https://doi.org/10.1371/journal.pone.0206792

${ }^{37}$ A. Baranda, art. cit. 
vulnerable dadas las desigualdades de género en el acceso a empleos de calidad en el sector formal y a la protección social. Las mujeres que encabezan hogares con hijos enfrentan el doble reto de proveer económicamente en su curso de vida y de generar un patrimonio que proteja a sus dependientes económicos en caso de ausencia. Si bien las trabajadoras formales tienen acceso a un seguro por invalidez y fallecimiento, los menores dependientes de las mujeres en el sector informal carecen de mecanismos de protección vinculados al empleo de la madre.

Según sus reglas de operación, el programa estaba orientado a "disminuir la condición de vulnerabilidad que enfrentan los hijos e hijas de hasta 23 años de edad en hogares con jefatura femenina con ingreso inferior a la línea de bienestar per cápita, ante el fallecimiento de sus madres”. Para ello, se estableció como población objetivo a los niños y jóvenes menores de 23 años, "en condición de orfandad materna, en cuyo hogar se haya tenido jefatura femenina en un rango de 12 a 68 años de edad, cuyo ingreso per cápita por hogar no rebasa la línea de bienestar". 38

El Seguro de Vida para Jefas de Familia consiste en una transferencia mensual para las hijas e hijos de hasta 23 años de edad en hogares con jefatura femenina, condicionada a su permanencia en el sistema educativo. El programa estableció montos diferenciados para las transferencias dependiendo del grado escolar de los menores beneficiarios, con cantidades mayores para quienes se hallen estudiando en los niveles medio superior y superior. ${ }^{39}$ Para las jefas de familia con

${ }^{38}$ Reglas de Operación del Programa Seguro de Vida para Jefas de Familia, para el ejercicio fiscal 2013, Diario Oficial de la Federación, 28 de febrero de 2013.

${ }^{39}$ Las cantidades se han ajustado a lo largo del tiempo. En 2013, los beneficiarios de 0 años a edad preescolar recibían 300 pesos mensuales y aquellos en educación superior 1000. En 2018, estos montos aumentaron a 330 y 1100 pesos respectivamente. El programa contemplaba transferencias de hasta 2040 pesos en 2018 para menores con necesidades especiales (montos de 2018). 
interés en el programa, se planteó un sistema de prerregistro mediante una solicitud para incorporarse, aunque esta petición no constituía "una obligación para la incorporación de los beneficiarios al programa, sino hasta que se cumplan los requisitos establecidos en las reglas de operación que se encuentren vigentes, es decir, acreditar la condición de vulnerabilidad o pobreza [...], al presentarse la condición de orfandad materna" ${ }^{40}$ En caso de fallecimiento de la madre y acreditación de los requisitos de incorporación, los beneficiarios deben presentar sus constancias de estudios de manera periódica para seguir recibiendo la transferencia.

La gráfica 5 muestra el número de jefas de familia prerregistradas en el programa en el periodo 2013-2018. Desde el inicio de la administración peñista, la Sedesol promovió la afiliación invitando a beneficiarias de programas existentes, como Oportunidades/Prospera, Guarderías y Estancias Infantiles y Seguro Popular. ${ }^{41}$ De 2013 a 2014, el número de prerregistros pasó de 3 millones a 5.5 millones. Dadas las características del programa, la cantidad de apoyos otorgados fue significativamente menor. En 2014, el presidente Peña Nieto informó que más de 1500 niñas y niños ya recibían el monto del seguro luego de la pérdida de su madre. ${ }^{42}$ Hacia el final del sexenio, el número de jefas de familia prerregistradas aumentó a 6.8 millones. Según la revisión de la Auditoría Superior de la Federación (ASF) correspondiente a 2017, la Sedesol reportó la entrega de subsidios a 40000 menores de edad en orfandad. ${ }^{43}$

${ }^{40}$ Reglas de Operación del Programa Seguro de Vida para Jefas de Familia para el ejercicio fiscal 2018, Diario Oficial de la Federación, 21 de diciembre de 2017. En caso de fallecimiento de la madre jefa de familia, se requiere a los tutores o hijos menores de 23 años acreditar la condición de vulnerabilidad económica del hogar de los beneficiarios y presentar la solicitud de prerregistro de la madre.

${ }^{41}$ Martha Martínez, "Apoyo a huérfanos, a cuentagotas", Reforma, 12 de junio de 2016.

42 "Peña Nieto anuncia plan...", art. cit.

43 Angélica Enciso, "La Sedesol incumple metas: ASF", La Jornada, 18 de septiembre de 2018. 
A pesar de que en el discurso del gobierno federal el Seguro de Vida se planteó como una política orientada a atender las necesidades de las mujeres en situación de vulnerabilidad, el diseño e implementación del programa reconoce como beneficiarios exclusivos a los hijos o hijas en edad escolar. En las reglas de operación del programa se señala que, para las personas en situación económica vulnerable, "la pérdida de los activos productivos que permiten a las personas generar ingresos para satisfacer sus necesidades básicas (como la pérdida del empleo o la pérdida del jefe o jefa de familia)" representa un riesgo.

Además, se menciona que en hogares con jefatura femenina, "al ser el único sustento familiar y al carecer de alguna red de protección social, en caso de pérdida de la madre, sus hijos e hijas pueden caer o empeorar su situación de pobreza, limitando así su desarrollo y oportunidades futuras". ${ }^{4}$ Por tanto, es posible afirmar que este programa no es una política orientada a reducir los obstáculos socioeconómicos que enfrentan las mujeres jefas de familia, pues está centrado exclusivamente en aquellos niños y jóvenes que ya experimentaron la trágica pérdida de su madre. ${ }^{45}$

A pesar de que la precariedad de los hogares con jefatura femenina es precisamente una de las justificaciones del programa, la política social derivada no atiende directamente

${ }^{44}$ Véase Reglas de Operación del Programa Seguro de Vida para Jefas de Familia, para el ejercicio fiscal 2013..., op. cit., "Introducción" (énfasis añadido).

${ }^{45}$ Es decir, por el diseño de este programa, ante la vulnerabilidad de las mujeres que encabezan hogares, la intervención del Estado ocurre sólo en el peor escenario: después del fallecimiento de la madre. Este criterio plantea preguntas importantes acerca de las prioridades de la agenda de política social en México. Un reto pendiente es que incorpore tanto las necesidades de las personas actualmente incorporadas a la fuerza de trabajo como las de las nuevas generaciones. Para una discusión sobre algunos efectos inesperados del diseño de programas sociales, véase Maxine Molyneux, "Mothers at the service of the new poverty agenda: Progresa/ Oportunidades, Mexico's conditional transfer programme", Social Policy Ẽ Administration, 40 (2006), pp. 425-449. 
esta vulnerabilidad. ${ }^{46} \mathrm{Si}$ bien el programa se presentó como una contribución a "la consolidación de un sistema de seguridad social universal, que es característica de un México incluyente", los alcances del programa no permiten categorizar las transferencias como una política orientada al logro de la seguridad social universal. ${ }^{47}$ Por ejemplo, uno de los cuestionamientos de organizaciones de derechos de la infancia fue que, considerando únicamente su objetivo de atender a los niños y jóvenes en situación de orfandad, el programa ha carecido de mecanismos de acompañamiento en salud. ${ }^{48}$

Según datos de la Iniciativa para el Fortalecimiento de la Institucionalidad de los Programas Sociales (IPRO), el monto asignado al programa Seguro de Vida pasó de alrededor de 400 millones de pesos en 2013 a 1000 millones en 2014, monto que se mantuvo relativamente estable hasta el presupuesto previsto para 2016. ${ }^{49}$ Para 2017, el presupuesto asignado se redujo significativamente y la fuente de financiamiento del programa pasó casi en su totalidad a un fideicomiso. ${ }^{50}$

${ }^{46}$ Este señalamiento ha estado presente en las percepciones de algunas beneficiarias. En una entrevista, una jefa de familia prerregistrada mencionó, en 2013, que si bien apreciaba el objetivo del programa, sería preferible que "el apoyo fuera en vida". Itxaro Arteta, "Atoran en Sedesol seguro para madres", Reforma, 31 de enero de 2013.

$47 \mathrm{Ibid}$. La meta "México incluyente" formó parte del Plan Nacional de Desarrollo 2013-2018.

${ }^{48}$ Itxaro Arteta, "Ven desarticulado seguro para huérfanos", Reforma, 16 marzo de 2013.

${ }^{49}$ Montos de gasto a precios corrientes. IPRo es un proyecto desarrollado por el Programa de Naciones Unidas para el Desarrollo y Transparencia Mexicana. Los datos reportados provienen de registros voluntarios de entidades federativas y dependencias federales de los programas sociales que están llevando a cabo. Véase IPRo, "Iniciativa para el fortalecimiento de la institucionalidad de los programas sociales", https://www.progra massociales.org.mx/

${ }^{50}$ Tania Rosas, "Reducen aportación a seguro para jefas de familia", $E l$ Economista, 26 de septiembre de 2016; Mathieu Tourliere, "Alerta Coneval por tijeretazo a programas para los más pobres", Proceso, 4 de octubre de 2016. 
GRÁFICA 5

Número de beneficiarias afiliadas al programa Seguro de Vida para Jefas de Familia (2013-2018)

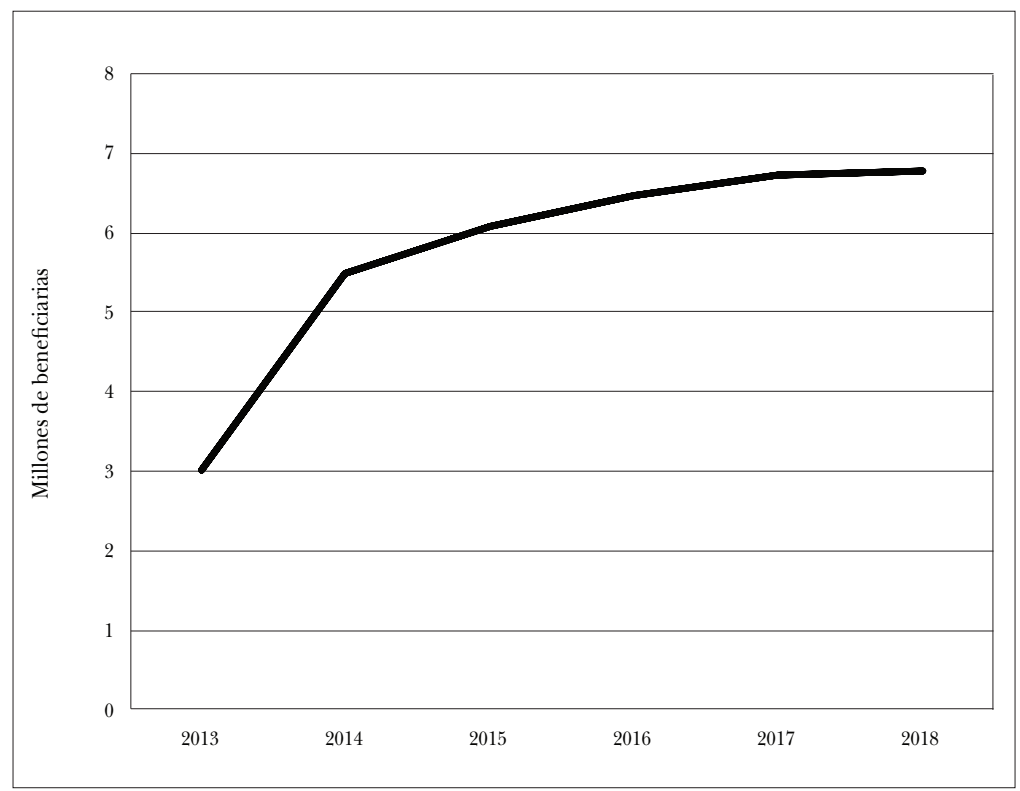

Fuente: elaboración propia con datos de la Secretaría de Desarrollo Social (Sedesol). Información obtenida vía IPRo, "Iniciativa para el fortalecimiento de la institucionalidad de los programas sociales", https://www. programassociales.org.mx/

Desde su creación, el Seguro de Vida enfrentó dificultades para ejercer los recursos asignados. Si bien se modificaron aspectos de la operación del programa para facilitar la afiliación y la entrega de recursos, hacia 2016 los indicadores de desempeño seguían mostrando un subejercicio crítico de los recursos disponibles. ${ }^{51}$ Además, los costos de operación del seguro se mantuvieron altos durante el sexenio, rebasando en algunos años los beneficios entregados a menores en

${ }^{51}$ M. Martínez, art. cit. 
orfandad. ${ }^{52}$ Después de su alta visibilidad durante los primeros años de la administración, el programa tuvo poca presencia en los discursos públicos del final del periodo del presidente Peña Nieto. El periódico Reforma reporta como último evento de alto perfil la entrega de apoyos el 10 de mayo de 2016, cuando se anunció la afiliación de 6000 mujeres policías al programa. ${ }^{53}$

\section{Conclusiones}

La agenda social de la administración de Peña Nieto tuvo como eje rector la estrategia Cruzada contra el Hambre. El énfasis en la reducción de la pobreza, según los criterios de este programa, prevaleció sobre otros objetivos, tales como el desarrollo de políticas sociales integrales para la reducción de las desigualdades de género. La falta de un enfoque en el curso de vida de las mujeres dificultó la vinculación entre los esfuerzos de política social. Además, el encuadre de los programas se acercó más a una lógica asistencial para la población vulnerable que a políticas propias de un Estado de bienestar, anclado en derechos sociales y compatible con un esquema universal para la ciudadanía en general.

Si bien el gobierno federal enfatizó la incorporación de un enfoque de género en el Plan Nacional de Desarrollo 2012-2018, ${ }^{54}$ la definición y características de este eje trans-

${ }^{52}$ En evaluaciones de Coneval y la ASF se señala que una deficiencia del programa con consecuencias en el ejercicio de los recursos ha sido la falta de información sobre los patrones de mortalidad de mujeres jefas de familia en hogares en situación económicamente vulnerable. Aunado a esto, en la revisión de la Cuenta Pública 2016 la ASF indicó que no era claro si se habían hecho proyecciones actuariales de gasto en apoyos individuales a largo plazo. Véase Claudia Salazar, "Alertan deficiencia en seguro de madres", Reforma, 2 de julio de 2017, y A. Enciso, art. cit.

${ }_{53}$ M. Martínez, art. cit.

${ }^{54}$ Gobierno de la República, Plan Nacional de Desarrollo 2013-2018, Diario Oficial de la Federación, 20 de mayo de 2013, http:/ / www.dof.gob. $\mathrm{mx} /$ nota_detalle.php?codigo $=5299465 \&$ fecha $=20 / 05 / 2013$ 
versal fueron limitadas. En la justificación de los programas se asoció lo femenino casi exclusivamente a la maternidad y a prioridades diferenciadas derivadas de la mayor carga de las mujeres en el trabajo doméstico y de cuidados. El Seguro para Jefas de Familia, por ejemplo, es un programa de apoyo orientado específicamente hacia menores en orfandad, pero se presentó como parte del esquema de políticas diseñadas en beneficio de mujeres económicamente vulnerables. Así, la reducción de la desigualdad de género en la protección social o la socialización de los cuidados quedaron como asignaturas pendientes.

Finalmente, el diseño e implementación de la agenda social del sexenio reafirmó la fragmentación en la provisión de las políticas sociales en México. En consonancia con administraciones anteriores, se privilegiaron los esquemas de transferencias y subrogación de servicios, lo cual implicó bajos niveles de institucionalidad en los esfuerzos sociales del gobierno federal. En términos de los tres programas analizados en este artículo, el truncamiento de la provisión de políticas sociales dificultó la formación de coaliciones sociales amplias para demandar y defender las acciones sociales del Estado mexicano.

\section{REFERENCIAS BIBLIOGRÁFICAS}

Acuerdo por el que se emiten las Reglas de Operación del Programa de Pensión para Adultos Mayores, para el ejercicio fiscal 2013, Diario Oficial de la Federación, 20 de febrero de 2013. Águila, Emma, Mariana LóPEz-Ortega y Luis Miguel GutiérRez Robledo ÁGuila, "Non-contributory pension programs and frailty of older adults: evidence from Mexico", PLos One (2018), núm. 11, https://doi.org/10.1371/journal.pone.0206792

Arteta, Itxaro, "Atoran en Sedesol seguro para madres", Reforma, 31 de enero de 2013.

Arteta, Itxaro, "Alinean proyecto con 65 y Más", Reforma, 19 de febrero de 2013. 
Arteta, Itxaro, "Ven desarticulado seguro para huérfanos", Reforma, 16 marzo de 2013.

Arteta, Itxaro, "Priorizan para 2014 programas sociales", Reforma, 10 de septiembre de 2013.

Auditoría Superior de la Federación, "Informe Individual del Resultado de la Fiscalización Superior de la Cuenta Pública 2017, Auditoría de Desempeño: 2017-0-20100-07-0265-2018”, 2017, https:/ /www.asf.gob.mx/Trans/Informes/IR2017a/Docu mentos/Auditorias/2017_0265_a.pdf

Baranda, Antonio, "Alista Peña Nieto plan antipobreza”, Reforma, 10 de enero de 2013.

Calderón, Gabriela, "The Effects of Child Care Provision in Mexico”, documento de trabajo 2014-07, Banco de México, 2014.

Cejudo, Guillermo M., Cynthia L. Michel y Roberto Gerhard, "Metaevaluación del Programa de Estancias Infantiles (PEI)", México, CIDE-CleAR, 2012.

Dion, Michelle, "Retrenchment and Reform," en Workers and welfare: Comparative institutional change in twentieth-century Mexico, Pittsburgh, University of Pittsburgh Press, 2010, pp. 116-151.

Enciso, Angélica, "La Sedesol incumple metas: AsF", La Jornada, 18 de septiembre de 2017.

Esping-Andersen, Gøsta, "A new gender contract”, en Gøsta Esping-Andersen (ed.), Why we need a new welfare state, Oxford, University Press, 2002.

Flamand, Laura, "La reforma perdida. Tendencias de la política social en México (2012-2015)", en Fernando Nieto y Ernesto Velasco (eds.), Una agenda para la administración pública: reconocimiento a la trayectoria de María del Carmen Pardo, México, El Colegio de México, 2018.

Flamand, Laura, y Carlos Moreno-Jaimes, "La protección social en salud durante el gobierno de Calderón. Avances y rezagos en el diseño y la implementación del Seguro Popular (20062012)", Foro Internacional (2015), núm. 1, pp. 217-261.

Fraser, Nancy, "After the Family Wage: Gender Equity and the Welfare State”, Political Theory, 22 (1994), pp. 591-618. 
García, Brígida, "El trabajo doméstico y de cuidado: su importancia y principales hallazgos en el caso mexicano", Estudios Demográficos y Urbanos, 34 (2019), pp. 237-267.

García, Brígida y Edith Pacheco (coords.), Uso del tiempo y trabajo no remunerado en México, México, El Colegio de México, 2014.

Gasparini, Leonardo y Mariana Marchionni, Bridging gender gaps? The rise and deceleration of female labor force participation in Latin America, La Plata, CEDLAs, 2015.

Gobierno de la República, Plan Nacional de Desarrollo 2013-2018, Diario Oficial de la Federación, 20 de mayo de 2013, http:// www.dof.gob.mx/nota_detalle.php?codigo $=5299465 \&$ \&ec ha $=20 / 05 / 2013$

Gobierno de México, Issste, "Estadística, Anuarios 2017", http:/ / www.issste.gob.mx/datosabiertos/anuarios/anuarios2017.html Gobierno de México, Imss, "Memoria Estadística 2017”, http:/ / www.imss.gob.mx/conoce-al-imss/memoria-estadistica-2017

González Marín, María Luisa, Patricia Rodríguez y Nadima SiMón Domínguez, "Las mujeres y los nuevos sistemas de pensiones en México", Revista de Investigación Social, 4 (2007), pp. 109-133.

Gornick, Janet C. y Marcia K. Meyers, "Creating gender egalitarian societies: An agenda for reform”, Politics Ev Society, 36 (2008), pp. 313-349.

Guerrero, Claudia, "Defienden los priistas cruzada contra hambre", Reforma, 12 de febrero de 2013.

Gutiérrez, Emilio, Laura JuÁrez y Adrián Rubli, "The Effect of a Transfer Program for the Elderly in Mexico City on Co-Residing Children's School Enrollment”, World Bank Economic Review, 31 (2017), pp. 809-828.

Instituto Nacional de Estadística y Geografía, INEGI, "Encuesta Nacional de Empleo", México, INEGI, mayo de 2019, https://www. inegi.org.mx/contenidos/saladeprensa/boletines/2019/ enoe_ie/enoe_ie2019_08.pdf

Instituto Nacional de estadística y Geografía, INEGI, "Indicadores de ocupación y empleo. Cifras oportunas durante diciembre de 2018”, México, INEGi, 22 de enero de 2019, https://www.inegi. 
org.mx/contenidos/saladeprensa/boletines/2019/iooe/ iooe2019_01.pdf

Iniciativa para el Fortalecimiento de la Institucionalidad de los Programas Sociales, IPRO, "Iniciativa para el fortalecimiento de la institucionalidad de los programas sociales", https://www.pro gramassociales.org.mx/

Instituto Nacional de Estadística y Geografía, INEGI, Mujeres y hombres en México 2018, México, INEgi, 2018.

Liu, Chia, Albert Esteve y Rocío Treviño, "Female-headed households and living conditions in Latin America", World Development, vol. 90 (2017), pp. 311-328.

Marchionni, Mariana, Pablo Gluzmann, Joaquín Serrano y Monserrat Bustelo, Participación laboral femenina, ¿qué explica las brechas entre países?, IDB-CEDLAs, Nueva York, 2019, http:/ /dx.doi. org/10.18235/0001512

Martínez, Martha, "Apoyo a huérfanos, a cuentagotas", Reforma, 12 de junio de 2016.

Morgan, Kimberly J., "Path shifting of the welfare state: Electoral competition and the expansion of work-family policies in Western Europe”, World Politics vol. 65, núm. 1 (2013), pp. 73-115.

Molyneux, Maxine, "Mothers at the service of the new poverty agenda: Progresa/Oportunidades, Mexico's conditional transfer programme", Social Policy E Administration, 40 (2006), pp. 425-449.

Novella, Rafael, Andrea Repetto, Carolina Robino y Graciana Rucci (eds.), Millennials en América Latina y el Caribe: ¿trabajar o estudiar? CEEY- Banco Interamericano de Desarrollo, 2018, http:/ /dx.doi.org/10.18235/0001410

Organisation for Economic Co-operation and Development, OECD, "OecD Family Database" (base de datos en línea), http://www. oecd.org/els/family/database.htm

Organisation for Economic Co-operation and Development, OECD, "OECD. Stats" (base de datos en línea), https://stats.oecd.org/ index.aspx?queryid $=54741$

Organización Internacional del Trabajo, ort. Presente y futuro de la protección social en América Latina y el Caribe, Lima, OIT Oficina Regional para América Latina y el Caribe, 2018. 
"Peña Nieto anuncia plan de vivienda para jefas de familia", Notimex, 9 de mayo de 2014.

Perry, Guillermo, E. William F. Maloney, Omar S. Arias, Pablo Fajnzylber, Andrew D. Mason y Jaime SaAvedra-Chanduvi, Informality: Exit and exclusion, Washington, DC, World Bank Publications, 2007.

Pribble, Jennifer, "Mujeres y bienestar: Un estudio comparativo de Chile y Uruguay", en Alma Idiart (ed.), Estado benefactor y políticas sociales: Historia, implementación y reforma de programas sociales en Argentina, Chile y Uruguay, Buenos Aires, Biblos, 2011, pp. 49-76.

Reglas de Operación del Programa de Guarderías y Estancias Infantiles para Apoyar a Madres Trabajadoras, para el ejercicio fiscal 2007, Diario Oficial de la Federación, 10 de enero de 2007.

Reglas de Operación del Programa Seguro de Vida para Jefas de Familia, para el ejercicio fiscal 2013, Diario Oficial de la Federación, 1 de marzo de 2013.

Rizzoli-Córdoba, Antonio, Laura Ibernia Vargas-Carrillo, Jorge Rodrigo Vásquez-Ríos, Hortensia Reyes-Morales, Miguel Ángel Villasís-Keeverd, Gabriel O'Shea-Cuevas, Daniel Aceves-Villagrán, Onofre Muñoz-Hernández y José Alberto García-Aranda, "Asociación entre el tiempo de permanencia en el Programa de Estancias Infantiles para niños en situación de pobreza y el nivel de desarrollo infantil”, Boletín Médico del Hospital Infantil de Meéxico (2017), núm. 2, pp. 98-106.

Rodin, Danielle L., Kristen McNeill, Norma Vite-Leon y Jody Heymann, "Determinants of informal employment among working mothers in Mexico", Community, Work Ev Family, 2012, núm. 1, pp. 85-99.

Rosas, Tania, "Reducen aportación a seguro para jefas de familia", El Economista, 26 de septiembre de 2016.

Sainsbury, Diane, Gender, Equality, and Welfare States, Nueva York, Cambridge University Press, 1996.

Salazar, Claudia, "Alertan deficiencia en seguro de madres", $R e-$ forma, 2 de julio de 2017.

SAlazAr, Claudia, "Acusan a la Sedesol de inflar presupuesto", $R e^{-}$ forma, 2 de noviembre de 2014. 
Tourliere, Mathieu, "Alerta Coneval por tijeretazo a programas para los más pobres”, Proceso, 4 de octubre de 2016.

Yunes, Miguel Ángel, La reforma del Issste: un cambio necesario, México, FCE, 2009. 
